\title{
HALOPERIDOL AND MIDAZOLAM DON’T WORK, AND NOW WHAT?
}

F. Godinho1, S. Escária²

${ }^{1}$ Hospital Espirito Santo de Évora, Department of Psychiatry and Mental Health, Évora, Portugal. ${ }^{1}$ Hospital Espirito Santo de Évora, Department of Internal Medicine, Évora, Portugal.

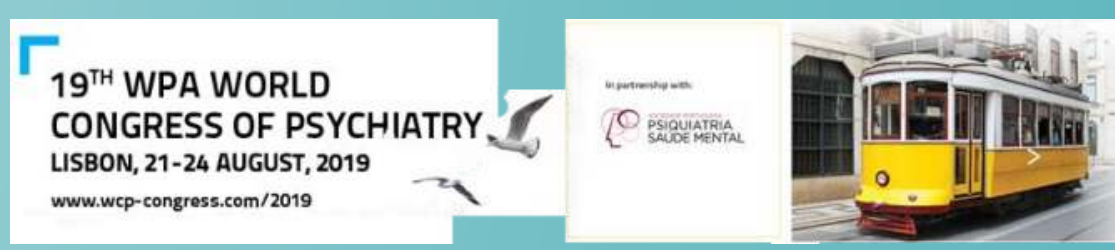

\section{Objective:}

To describe a case of successful management of refractory agitation with dexmedetomidine in the emergency department.

\section{Background:}

- Agitation in the emergency department is frequent, and its correct approach is fundamental to identify and treat its etiology.

- Regardless of the cause, emergency psychiatrist is frequently called.

- Suggested pharmacological approaches to agitation include antipsychotics and benzodiazepines.

\section{Materials and methods:}

Case report and brief review of dexmedetomidine.

\section{RESULTS}

\section{Clinical case}

65 years old female,

Psychiatric history of persistent depressive disorder

No previous medical history. No drugs or alcohol consumption history

Brought to the ER due to vomiting and altered behavior with severe agitation and suspected meningitis.

Chlorpromazine IM until 75 mg was administered, with no effect.

Emergency psychiatrist was called $\square$ olanzapine $\mathbf{2 0} \mathbf{~ m g}$ and Haloperidol $\mathbf{1 0} \mathbf{~ m g ~ I M}$ was also administered with no effect.

Due to persistent agitation: midazolam was started, until $\mathbf{1 8 m g}$, with respiratory depression and agitation recurrence when stopped.

The decision of starting dexmedetomidine perfusion was then made, with correct management of agitation, and making possible therapeutic procedures.

\section{Dexmedetomidine}

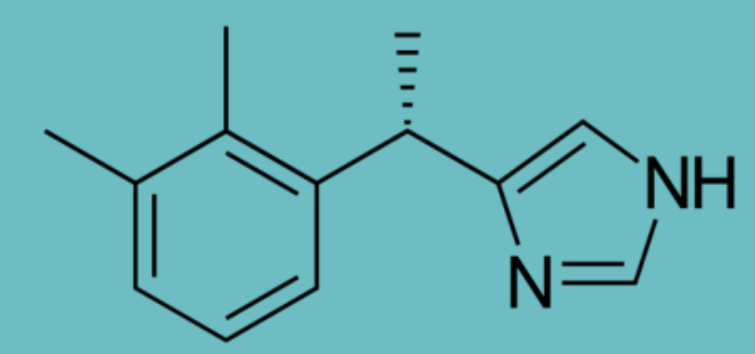

Dexmedetomidine is a selective alpha-2 receptor agonist.

Mechanism of action: Stimulation of parasympathetic outflow and inhibition of sympathetic outflow from the locus coeruleus. Higher $\alpha 2$ affinity when compared to clonidine.

Indication (EMA): for sedation of adult patients in Intensive Care Unit and for sedation of non-intubated adult patients prior to and/or during diagnostic or surgical procedures requiring sedation.

Recent clinical studies compare the use of Dexmedetomidine with other classical agents used to treat delirium (p.e. haloperidol) in non-intubated patients with promising results.
$\mathrm{Ng}$ KT
(2019)
Meta-analysis was reduced, odds ratio $(95 \% \mathrm{Cl}) 0.36(0.26-0.51), p<0.001$ "
"The use of dexmedetomidine was associated with a reduced incidence of agitation, OR $(95 \% \mathrm{Cl}) 0.34(0.20-0.59), p<0.001^{\prime \prime}$

"In the patients who received dexmedetomidine (eight trials, 1425 patients), delirium

Carrasco G NonRCT vs "dexmedetomidine shows to be useful as a rescue drug for treating agitation (2016) Haloperidol due to delirium in nonintubated patients in whom haloperidol has failed"

\section{Conclusion:}

Dexmetedomidine is mainly used in the ICU context, however, because its sedative effects and no significant respiratory depression, there is potential for other uses.

Further studies in non-intubated patients are necessary for the safe and effective use of this drug in the emergency context.

\section{References:}

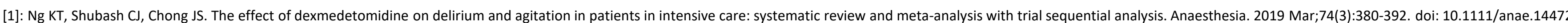

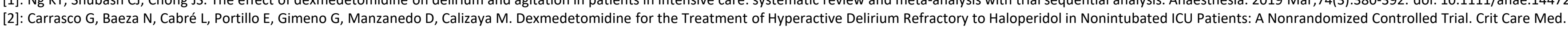
2016 Jul;44(7):1295-306. doi: 10.1097/CCM.0000000000001622. 